Published in final edited form as:

J Am Chem Soc. 2016 July 27; 138(29): 9269-9276. doi:10.1021/jacs.6b04966.

\title{
Ligand-Promoted Meta-C-H Arylation of Anilines, Phenols, and Heterocycles
}

\author{
Peng Wang ${ }^{\dagger,}{ }^{\perp}$, Marcus E. Farmer ${ }^{\dagger,}{ }^{\perp}$, Xing Huo ${ }^{\dagger},^{\perp}$, Pankaj Jain ${ }^{\dagger}$, Peng-Xiang Shen ${ }^{\dagger}$, Mette \\ Ishoey ${ }^{\ddagger}$, James E. Bradner ${ }^{\ddagger}$, Steven R. Wisniewski§, Martin D. Eastgate§, and Jin-Quan \\ $\mathrm{Yu}^{*}, \dagger$ \\ †Department of Chemistry, The Scripps Research Institute, 10550 N. Torrey Pines Road, La Jolla, \\ California 92037 \\ ¥Department of Medical Oncology, Dana-Farber Cancer Institute, 360 Longwood Ave. Boston, MA \\ 02115, USA \\ $\S$ Chemical Development, Bristol-Myers Squibb, 1 Squibb Drive, New Brunswick, New Jersey \\ 08903, USA
}

\begin{abstract}
Here we report the development of a versatile 3-acetylamino-2-hydroxypyridine class of ligands that promote meta-C-H arylation of anilines, heterocyclic aromatic amines, phenols, and 2-benzyl heterocycles using norbornene as a transient mediator. More than 120 examples are presented, demonstrating this ligand scaffold enables a wide substrate and coupling partner scope. Meta-C-H arylation with heterocyclic aryl iodides as coupling partners is also realized for the first time using this ligand. The utility for this transformation for drug discovery is showcased by allowing the meta-C-H arylation of a lenalidomide derivative. The first steps towards a silver free protocol for this reaction are also demonstrated.
\end{abstract}

\section{TOC Graphic}
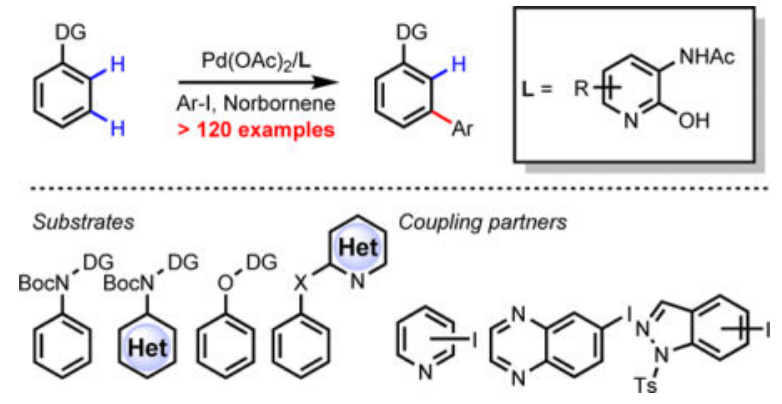

\footnotetext{
*Corresponding author. Yu200@scripps.edu.

Author Contributions. P.W, M.E.F., \& X.H. contributed equally to this work.
}

Supporting Information Available. Detailed experimental procedures, characterization of new compounds. This material is available free of charge via the internet at http://pubs.acs.org.

Notes. The authors declare no competing financial interest. 


\section{Introduction}

Cyclometallation has been extensively studied since the 1960s when the first examples of such process were disclosed. ${ }^{1}$ These initial reports spurred interest in the catalytic activation and functionalization of $\mathrm{C}-\mathrm{H}$ bonds directed by both installed and native functionality within a given molecule. In the last two decades, directed $\mathrm{C}-\mathrm{H}$ activation has gained significant traction and robust ortho-functionalizations of arenes using this strategy have been reported. ${ }^{2}$ In sharp contrast, the development of meta- and para-C-H functionalizations has seen much less success. Thus far, two approaches towards the selective meta- and para$\mathrm{C}-\mathrm{H}$ functionalization of arenes have predominated in the literature. The first strategy is the use of sterics and electronics to guide transition metal catalysts to the meta- or para-C-H bond of a given substrate. ${ }^{3}$ This strategy is the longest standing approach to this problem, though it is largely limited to 1,3-disubstituted arenes. ${ }^{3 c-e}$ The second, inspired by the use of directing groups to carefully guide transition metal catalysts, relies on the use of a template to guide a palladium catalyst to the remote meta- or para-C-H bond. ${ }^{4}$ Particularly for meta$\mathrm{C}-\mathrm{H}$ functionalization, this approach has been shown to be highly general. However, a downside to this approach is that the use of a designer template is required for each substrate class. A third and thought provoking strategy using $\mathrm{Ru}(\mathrm{II})$ catalysts has recently gained attention and holds significant promise if the substrate scope can be expanded to a wide range of directing groups. ${ }^{5}$ This approach hinges on the use of an initial ortho-

cycloruthenation which activates the meta-position (para to the newly formed C-Ru bond) of an aromatic ring to react with electrophiles. ${ }^{5}$ The mechanism of this transformation is somewhat unclear, though a radical pathway has recently been proposed. ${ }^{5 \mathrm{c}}$ Several other approaches towards the meta- $\mathrm{C}-\mathrm{H}$ functionalization of aromatics have also been demonstrated, though the scope and generality of these approaches remains to be fully explored. ${ }^{6}$

Recently, our group and others have developed another approach to achieve meta-C-H functionalization by relaying the initial ortho-cyclopalladation to the meta-position using norbornene as the mediator. ${ }^{7}$ Such a relay process is the key step in the well documented Catellani reaction. ${ }^{8}$ In this reaction, norbornene acts as an efficient mediator that relays palladium from an initial site of palladation (obtained via oxidative addition of $\operatorname{Pd}(0)$ to an aryl halide) to the adjacent position. ${ }^{8}$ In an interesting example, Bach has also shown that the use of norbornene as a transient mediator can be successfully combined with palladium catalyzed 1,2-aminopalladation to achieve selective $\mathrm{C}-\mathrm{H}$ functionalization at the 2-position of indoles and pyrroles. ${ }^{9}$ Though the Catellani reaction has been known since 1997, the use of norbornene as a transient mediator had not been successfully combined with directed ortho-C-H activation to achieve a net meta-functionalization via a relay process until 2015 (for a simplified mechanism, see Figure 1a). ${ }^{7}$ Though this strategy in theory should be able to translate the plethora of ortho-C-H activation reactions to meta- $\mathrm{C}-\mathrm{H}$ functionalization, currently only a handful of substrates have been demonstrated to be compatible with this catalysis. ${ }^{7}$ Notably, to the best of our knowledge, there have been no reports disclosing the use of heterocyclic aryl iodides as coupling partners in this transformation. Further development of this newly emerging meta-C-H functionalization strategy remains an important challenge as it holds the potential to be a very general approach towards the meta- 
$\mathrm{C}-\mathrm{H}$ functionalization of aromatics in a reliable and predictable manner. To overcome the current limitations of this approach, the identification of versatile ligands that can promote this transformation is necessary. Specifically, ligands need to be developed which can work in concert with a wide range of directing groups to promote the $\mathrm{C}-\mathrm{H}$ activation and subsequent functionalization steps while disfavoring potential side reactions. Herein, we report the identification of highly versatile 3-acetylamino-2-hydroxypyridine ligands which promote the meta-C-H arylation of anilines, heterocyclic aromatic amines, phenols, and 2benzyl heterocycles using norbornene as a transient mediator. These ligands enable a broad substrate scope for this transformation and allow a variety of heterocyclic aryl iodides to be used as effective coupling partners.

\section{Results and Discussion}

In an attempt to expand the scope of norbornene-mediated meta- $\mathrm{C}-\mathrm{H}$ arylation reactions, we selected aniline as a model substrate. Meta-functionalization of this class of compounds $4 \mathrm{~b}, 5 \mathrm{c}, 6 \mathrm{~d}, \mathrm{e}$ is highly valuable as electrophilic aromatic substitution reactions predominantly afford ortho- and/or para-substituted products due to the electronic effects of the nitrogen atom. Our initial evaluation of aniline substrates focused on finding a reactive and readily removable directing group that would be compatible with the desired catalytic cycle. After a survey of several directing groups, we found that a benzylicpyridine based directing group allowed formation of the meta-arylated product in 13\% yield while other directing groups were not reactive (see supporting information for full screening details). Such directing groups that promote the formation 7-membered palladacycles are uncommon; however, the use of similar directing groups is precedented in $\mathrm{Pd}(\mathrm{II})$ catalyzed $\mathrm{C}-\mathrm{H}$ olefination of phenols, anilines and benzylamines. ${ }^{10} \mathrm{Next}$, we attempted to identify ligands that could match with this directing group and promote the reaction, starting with both amino acid and pyridine or quinoline derived ligands which have been shown to promote $\mathrm{C}-\mathrm{H}$ activation reactions $\mathrm{s}^{4,7 \mathrm{a}, \mathrm{c}}$ (Table 1 ). Intriguingly, only a minimal improvement of the yield was obtained when utilizing either of these previously established ligand classes. Given the enabling role of the pyridine derived ligand $\mathbf{L 1}$ in the norbornene-mediated meta$\mathrm{C}-\mathrm{H}$ arylation of phenylacetic amide derived substrates previously reported by our laboratory ${ }^{7 \mathrm{a}, \mathrm{c}}$, this result implies that new ligand scaffolds must be discovered to extend to other classes of substrates. Phosphine (L4 and L5) and N-heterocyclic carbene (L6) ligands were also evaluated, but neither provided a significant improvement of the yield. At this stage, the notion that the multiple steps of this complex catalytic cycle may require different ligand coordination turned our attention to 2-pyridone based ligands. These ligands can coordinate as either a $\sigma$-donor through the pyridyl nitrogen or a $\kappa^{2}$ anion analogous to carboxylates. When using 2-pyridone as the ligand a slight improvement in the yield was obtained. Interestingly, substitution of the 3-position on this scaffold with an NHAc moiety (L12) provided a significant improvement to the reaction, affording the desired product in $42 \%$ yield with good mass balance (Table 1). This substitution was chosen as we hypothesized that installation of this NHAc provides a secondary binding site that may serve a functional role in catalysis. Bisdentate coordination between the NAc and the oxygen atom would form a structure that is reminiscent of mono-protected amino acid (MPAA) ligands developed by our laboratory for $\mathrm{C}-\mathrm{H}$ functionalization reactions ${ }^{11}$ (Figure 1.). An important 
feature of these newly disclosed ligands is their modularity. By appropriate choice of substitution on the aromatic ring, one can readily tune the coordination strength of this ligand scaffold to the metal catalyst which in turn should allow this class of ligands to be easily adjusted to match with a variety of substrates.

Given the importance of synergy between directing group and ligand for efficient $\mathrm{C}-\mathrm{H}$ functionalization ${ }^{12}$, we re-visited the directing groups and found that electron rich benzylic pyridine based directing groups matched well with this ligand scaffold. A removable, commercially available directing group (DG, figure 2, see supporting information for DG screening and removal) was found to provide optimal synergy with the ligand allowing formation of the desired product in $98 \%$ isolated yield. With the final optimized reaction conditions in hand, we set out to evaluate the substrate scope of the reaction. As can be seen in table 2, the substrate scope of this ligand promoted transformation is broad. Substrates bearing electron-donating and electron-withdrawing groups (1a-0) at either the ortho- or meta-positions of the aniline react smoothly providing the desired products in greater than $70 \%$ yield. Note that the highly electron-withdrawing trifluoromethyl, cyano and nitro functionalities $(\mathbf{5 g}, \mathbf{5 j}, \mathbf{5 k})$ are tolerated in this reaction when utilizing an improved transient mediator, methyl bicyclo[2.2.1] hept-2-ene-2-carboxylate $\left(\mathrm{NBE}-\mathrm{CO}_{2} \mathrm{Me}\right)$, in place of 2norbornene. ${ }^{7 \mathrm{c}}$ Interestingly, unsubstituted aniline $\mathbf{1 l}$ and para-substituted substrate $\mathbf{1 p}$ showed high selectivity for the di-substituted product, whereas 4-methoxy substituted aniline 1q showed high mono-selectivity. Currently, we hypothesize that after the initial arylation of substrate 1q, a conformational change is induced wherein the methyl group on the methoxy is primarily positioned away from the newly installed aryl ring, sterically hindering the alternative meta-position which prevents di-arylation. Heterocyclic substrates (2a-l), which are commonly incompatible with $\mathrm{C}-\mathrm{H}$ cross-coupling methodology as they poison the transition metal catalyst, react in good to excellent yields forming products $\mathbf{6 a - 1}$. Importantly, the yield of product $\mathbf{6 b}$ was reduced to $4 \%$ in the absence of $\mathbf{L 1 2}$ (table 2) thus indicating the importance of the ligand to achieve a broad substrate scope for this transformation.

Interested in further examining the breadth of this reaction, we next evaluated meta-C-H arylation of phenol ${ }^{4 \mathrm{c}, 6 \mathrm{f}}$ derived substrates bearing benzylic pyridine based directing groups. ${ }^{10 a}$ Though good yields were obtained with the same directing group that was utilized for the aniline derived substrates, deprotection proved problematic. To circumvent this problem, we opted to use a 2,3-lutidine derived directing group $\left(\mathrm{DG}^{\prime}\right)$ which is removed by hydrogenolysis with catalytic palladium on carbon under pressurized hydrogen (see supporting information for directing group removal). As shown in figure 2, a variety of phenols could be successfully arylated at the meta-position utilizing $\mathrm{NBE}-\mathrm{CO}_{2} \mathrm{Me}$ and a modified ligand (L14). Intriguingly, though substrate 11 shows high selectivity for diarylated product, phenolic substrate $\mathbf{3 d}$ provides a mono:di ratio of $1: 1$. We currently attribute this to the phenolic substrates being slightly less reactive than their aniline counterparts. To fully explore the scope of this methodology, we evaluated how this ligand would match with substrates containing native heterocycles as directing groups.

Gratifyingly, heterocyclic substrates that form 6-membered palladacycles ${ }^{13}$ upon cyclopalladation worked exceedingly well in the presence of $\mathbf{L 1 4}$. The ability of these 
ligands to promote this catalysis with directing groups that form 6- and 7-membered palladacycles indicates that they are potentially applicable to $\mathrm{Pd}(\mathrm{II})$ catalyzed meta-C-H functionalizations of substrates beyond the scope reported in this article. Substrates directed by native pyridine, pyrimidine, pyrazine, pyrazole, indazole, isoindazole and isoquinoline were all successfully arylated at the previously difficult-to-access meta-positions. As all of these substrates are unsubstituted it was interesting that significant variations in mono:di ratios were observed. We currently hypothesize that these ratios are related to the relative rates of cyclometallation and substrate exchange on the palladium catalyst. An in-depth study of the cyclometallation kinetics under these reaction conditions is required to confirm this hypothesis. ${ }^{13}$

Having thoroughly examined the substrate scope of this reaction, we turned our focus to evaluating the coupling partner scope. We choose 1a as the model substrate to investigate the reactivity of a wide range of aryl iodides. Experimental results show that this reaction possesses an exceptionally broad coupling partner scope when utilizing $\mathrm{NBE}-\mathrm{CO}_{2} \mathrm{Me} .^{7 \mathrm{c}}$ Electron donating and electron withdrawing groups at the para- and meta-positions of the aryl iodide coupling partner were well tolerated, providing the desired products in good yields $(\mathbf{9 a}-\mathbf{y})$. Interestingly, this reaction was not limited to simple aryl iodides as an array of heterocyclic aryl iodides was found to work well (9ae-ba). Indoles, thiophenes, furan, indazole, quinoline, quinazoline, and a range of pyridines were suitable coupling partners. To determine whether the broad coupling partner scope observed in this reaction was enabled by the ligand, we attempted the reaction under the optimized conditions with 2chloro-4-iodopyridine in the absence of $\mathbf{L 1 2}$ and found the yield to be $9 \%$ by ${ }^{1} \mathrm{H}$ NMR. This result highlights the importance of the ligands to achieve a broad coupling partner scope. In order to fully investigate the compatibility of this reaction with heterocycles, we explored the efficiency of coupling heterocyclic substrates with heterocyclic aryl iodides. As can be seen in table 3 (9bb-9bh), this ligand enables the coupling of heterocyclic coupling partners with heterocyclic substrates in reasonable to excellent yields. Furthermore, the utility of this reaction on a preparative scale was demonstrated by performing the meta-C- $\mathrm{H}$ coupling of aniline substrate 1a with methyl 4-iodobenzoate on gram-scale with $5 \mathrm{~mol} \% \mathrm{Pd}(\mathrm{OAc})_{2}$ and $5 \mathrm{~mol} \% \mathbf{~ L 1 2}$, affording the desired product (9n, see supporting information) cleanly in 93\% yield.

Having examined the efficiency of the meta- $\mathrm{C}-\mathrm{H}$ coupling of aniline substrates utilizing 3acetylamino-2-hydroxypyridine based ligands, we set out to demonstrate the utility of this methodology by applying it to the meta-arylation of a lenalidomide derivative. Recently, the drug thalidomide and its derivatives pomalidomide and lenalidomide have been repurposed for several clinical indications including multiple myeloma and myelodysplasia. Meta-C-H arylation of this scaffold exemplifies a scenario where utilizing norbornene mediated meta$\mathrm{C}-\mathrm{H}$ functionalization is advantageous as it allows for elaboration of the parent drug molecule in relatively few steps. Gratifyingly, the meta-arylation of a lenalidomide derivative proceeded smoothly to provide the desired product in $61 \%$ isolated yield (Scheme 1a). The successful application of the norbornene mediated meta-C-H functionalization in this setting showcases the potential of this reaction to be applied in drug discovery. 
In collaboration with Bristol-Myers Squibb, we set out to improve the practicality of the reaction conditions to enable this methodology to be used in the pharmaceutical industry. A high throughput screen was undertaken to establish Ag-free conditions with pharmaceutical process-friendly solvents. It was discovered that use of CsOAc in place of AgOAc in $t$ Amyl-OH could give synthetically useful yields with ortho-substituted aryl iodides on gram scale using $5 \mathrm{~mol} \% \mathrm{Pd}(\mathrm{OAc})_{2}$ utilizing $\mathbf{L 1 2}$ as the ligand. Following an iterative procedure (Scheme 1b), 73\% yield of 9a can be obtained when using $\mathbf{L 1 7}$ as the ligand. The removal of silver from the $\mathrm{Pd}(\mathrm{II})$-catalyzed, norbornene mediated meta-C-H arylation reaction will prove crucial for adopting this method in synthesis, especially when reactions need to be performed beyond gram-scale as stoichiometric use of this metal can become cost prohibitive.

The effectiveness of this ligand scaffold at providing a broad substrate and coupling partner scope for this transformation prompted us to investigate the nature by which it operates. Given the drastic improvement in yield obtained by the installation of an NHAc on the ligand scaffold in our early investigations (see table 1), we became curious as to its role in this catalytic manifold. Our original hypothesis was that this ligand may be bifunctional in nature and that coordination to the oxygen and the NHAc in a bidentate fashion may result in the formation of an active catalytic species (Figure 2). This hypothesis was formed due to analogy of this coordination mode with that of the NHAc variants of mono-protected amino acid (MPAA) ligands commonly employed by our group. ${ }^{11}$ To evaluate an analogous binding mode in our new ligand, a re-evaluation of 2-hydroxypyridine/2-pyridone using the optimal conditions was undertaken to shed light on the role of the NHAc moiety on the ligand. Interestingly, this study revealed that 2-pyridone could also promote this reaction under the fully optimized conditions. However, it is evident that the NHAc plays an instrumental role in enabling meta-C-H arylation involving difficult substrates and coupling partners (i.e. those containing heterocycles). For example, the yields of $\mathbf{6 b}$ and 9 as (see supporting information) were $40 \%$ and $65 \%$ respectively when using 2-pyridone as the ligand, whereas the yields when using $\mathbf{L 1 2}$ were $72 \%$ and $96 \%$ respectively. These results indicate that removal of the NHAc from the ligand under the fully optimized conditions does not completely ablate the reactivity of this scaffold, though it does result in a decrease in reactivity. A full mechanistic inquiry into the enabling nature of the ligands disclosed in this paper is underway. The results of this investigation, as well as their implication to improved catalysts/ligands, will be reported in due course.

\section{Conclusion}

In summary, a versatile ligand scaffold for Pd(II) catalyzed meta-C-H arylation of a wide range of arenes using norbornene as a transient mediator has been disclosed. Heterocyclic substrates and heterocycle-containing coupling partners are well tolerated under the developed reaction conditions, which should enable rapid uptake of this ligand enabled methodology in drug discovery. Exemplary of the potential for this reaction to be utilized in drug discovery, a lenalidomide derivative was smoothly arylated at the meta-C-H bond. The first example of a silver free protocol for this catalytic manifold has also been demonstrated. Further improvement in the efficiency of a silver free protocol for this reaction will aid in enabling practical applications of this methodology. A thorough investigation into the nature 
of these ligands, as well as their application to other transformations will be disclosed shortly.

\section{Experimental Section}

\section{General procedure for the ligand-promoted norbornene-mediated meta-C-H activation of anilines}

Substrate (0.1 mmol), Ar-I (0.2 mmol), Pd(OAc) 2 (2.2 mg, $10 \mathrm{~mol} \%), \mathbf{L 1 2}(3.0 \mathrm{mg}, 20 \mathrm{~mol}$ $\%$ ), AgOAc (50.1 mg, $0.3 \mathrm{mmol}), 2$-norbornene (14.1 mg, $0.15 \mathrm{mmol}$ ) or $\mathrm{NBE}^{-\mathrm{CO}_{2} \mathrm{Me}}$ $(21.6 \mathrm{mg}, 0.15 \mathrm{mmol})$ and 1,2-dichloroethane $(0.5 \mathrm{~mL})$ were added to a 2-dram vial. The vial was capped and closed tightly, then the reaction mixture was stirred at $100{ }^{\circ} \mathrm{C}$ for $24 \mathrm{~h}$. After cooling to room temperature, the mixture was passed through a pad of Celite with dichloromethane as the eluent to remove the insoluble precipitate. The resulting solution was concentrated and purified by preparative TLC to afford the desired arylated product. Full experimental details and characterization of new compounds can be found in the Supplementary Information.

\section{Supplementary Material}

Refer to Web version on PubMed Central for supplementary material.

\section{Acknowledgments}

We gratefully acknowledge The Scripps Research Institute and the NIH (NIGMS, 1R01 GM102265) for financial support.

\section{References}

1. a) Kleiman JP, Dubeck M. J Am Chem Soc. 1963; 85:1544.b) Cope AC, Siekman RW. J Am Chem Soc. $1965 ; 87: 3272$.

2. For selected reviews on Pd catalyzed C-H activation, see:a) Chen X, Engle KM, Wang DH, Yu JQ. Angew Chem Int Ed. 2009; 48:5094.b) Lyons TW, Sanford MS. Chem Rev. 2010; 110:1147. [PubMed: 20078038] c) Daugulis O, Do HQ, Shabashov D. Acc Chem Res. 2009; 42:1074. [PubMed: 19552413] For a review on Rh catalyzed C-H activation, see:d) Colby DA, Bergman RG, Ellman JA. Chem Rev. 2010; 110:624. [PubMed: 19438203] For a review on Ru catalyzed C-H activation, see:e) Arockiam PB, Bruneau C, Dixneuf PH. Chem Rev. 2012; 112:879.

3. a) Mkhalid IAI, Barnard JH, Marder TB, Murphy JM, Hartwig JF. Chem Rev. 2010; 110:890. [PubMed: 20028025] b) Maleczka RE Jr, Shi F, Holmes D, Smith MR III. J Am Chem Soc. 2003; 125:7792. [PubMed: 12822984] For examples of regioselective $\mathrm{C}-\mathrm{H}$ metallation without the need for 1,3-disubstitution, see:c) Cheng C, Hartwig JF. Science. 2014; 343:853. [PubMed: 24558154] d) Saito Y, Segawa Y, Itami K. J Am Chem Soc. 2015; 137:5193. [PubMed: 25860511] e) Kuninobu Y, Ida H, Nishi M, Kanai M. Nat Chem. 2015; 7:712. [PubMed: 26291942]

4. For select examples of template directed meta-C-H functionalization, see:a) Leow D, Li G, Mei TS, Yu JQ. Nature. 2012; 486:518. [PubMed: 22739317] b) Tang R, Li G, Yu JQ. Nature. 2014; 507:215. [PubMed: 24622200] c) Wan L, Dastbaravardeh N, Li G, Yu JQ. J Am Chem Soc. 2013; 135:18056. [PubMed: 24236533] For examples of template directed para-C-H functionalization, see:d) Bag S, Patra T, Modak A, Deb A, Maity S, Dutta U, Dey A, Kancherla R, Maji A, Hazra A, Bera M, Maiti DJ. Am Chem Soc. 2015; 137:11888.e) Patra T, Bag S, Kancherla R, Mondal A, Dey A, Pimparkar S, Agasti S, Modak A, Maiti D. Angew Chem Int Ed.

5. For examples of $\mathrm{Ru}(\mathrm{II})$ catalyzed meta-C-H functionalization via ortho-cyclometallation, see:a) Saidi O, Marafie J, Ledger AEW, Liu PM, Mahon MF, Kociok-Köhn G, Whittlesey MK, Frost CG. 
J Am Chem Soc. 2011; 133:19298. [PubMed: 22047022] b) Hofmann N, Ackermann L. J Am Chem Soc. 2013; 135:5877. [PubMed: 23534668] c) Li J, Warratz S, Zell D, De Sarkar S, Ishikawa EE, Ackermann L. J Am Chem Soc. 2015; 137:13894. [PubMed: 26418891] d) Teskey CJ, Lui AYW, Greaney MF. Angew Chem Int Ed. 2015; 54:11677.e) Paterson AJ, St John-Campbell S, Mahon MF, Press NJ, Frost CG. Chem Comm. 2015; 51:12807. [PubMed: 26166441]

6. For selected examples using copper and aryl iodoniums to achieve meta-C-H arylation, see:d) Phipps RJ, Gaunt MJ. Science. 2009; 323:1593. [PubMed: 19299616] e) Duong HA, Gilligan RE, Cooke ML, Phipps RJ, Gaunt MJ. Angew Chem Int Ed. 2010; 50:463.For an example of using $\mathrm{CO}_{2}$ as a traceless directing group, see:f) Luo J, Preciado S, Larrosa I. J Am Chem Soc. 2013; 136:4109.Kumar PNY, Bechtoldt A, Raghuvanshi K, Ackermann L. Angew Chem Int Ed. 2016; 55:6929.For an example using deprotonation, see:Martinez-Martinez AJ, Kennedy AR, Mulvey RE, O’Hara CT. Science. 2014; 346:834. [PubMed: 25395533]

7. a) Wang XC, Gong W, Fang LZ, Zhu RY, Li S, Engle KM, Yu JQ. Nature. 2015; 519:334. [PubMed: 25754328] b) Dong Z, Wang J, Dong G. J Am Chem Soc. 2015; 137:5887. [PubMed: 25909445] c) Shen PX, Wang XC, Wang P, Zhu RY, Yu JQ. J Am Chem Soc. 2015; 137:11574. [PubMed: 26313012] d) Han J, Zhang L, Zhu Y, Zheng Y, Chen X, Huang ZB, Shi DQ, Zhao Y. Chem Comm. 2016; 52:6903. [PubMed: 27142086]

8. a) Catellani M, Frignani F, Rangoni A. Angew Chem Int Ed. 1997; 36:119.For a review on norbornene mediated transformations, see:b) Ye J, Lautens M. Nature Chemistry. 2015; 7:863.

9. a) Jiao L, Bach T. J Am Chem Soc. 2011; 133:12990. [PubMed: 21806073] b) Jiao L, Herdtweck E, Bach T. J Am Chem Soc. 2012; 134:14563. [PubMed: 22913367] c) Jiao L, Bach T. Synthesis. 2014; 46:35-41.

10. a) Cong X, You J, Gao G, Lan J. Chem Comm. 2013; 49:662. [PubMed: 23146996] b) GarcíaRubia A, Urones B, Arrayás RG, Carretero JC. Angew Chem Int Ed. 2011; 50:10927.

11. a) Wang DH, Engle KM, Shi BF, Yu JQ. Science. 2010; 327:315. [PubMed: 19965380] b) Engle KM, Wang DH, Yu JQ. J Am Chem Soc. 2010; 132:14137. [PubMed: 20853838] c) Musaev DG, Kaledinm AL, Shi BF, Yu JQ. J Am Chem Soc. 2012; 134:1690. [PubMed: 22148424] d) Cheng, Gu-J., Yang, Y-F., Liu, P., Chen, P., Sun, T-Y., Li, G., Zhang, X., Houk, KN., Yu, J-Q., Wu, Y-D. J Am Chem Soc. 2014; 136:894. [PubMed: 24410499]

12. Engle KM, Yu JQ. J Org Chem. 2013; 78:8927. [PubMed: 23565982]

13. Desai LV, Stowers KJ, Sanford MS. J Am Chem Soc. 2008; 130:13285. [PubMed: 18781752] 
a. Simplified mechanism of norbornene mediated meta-C-H arylation

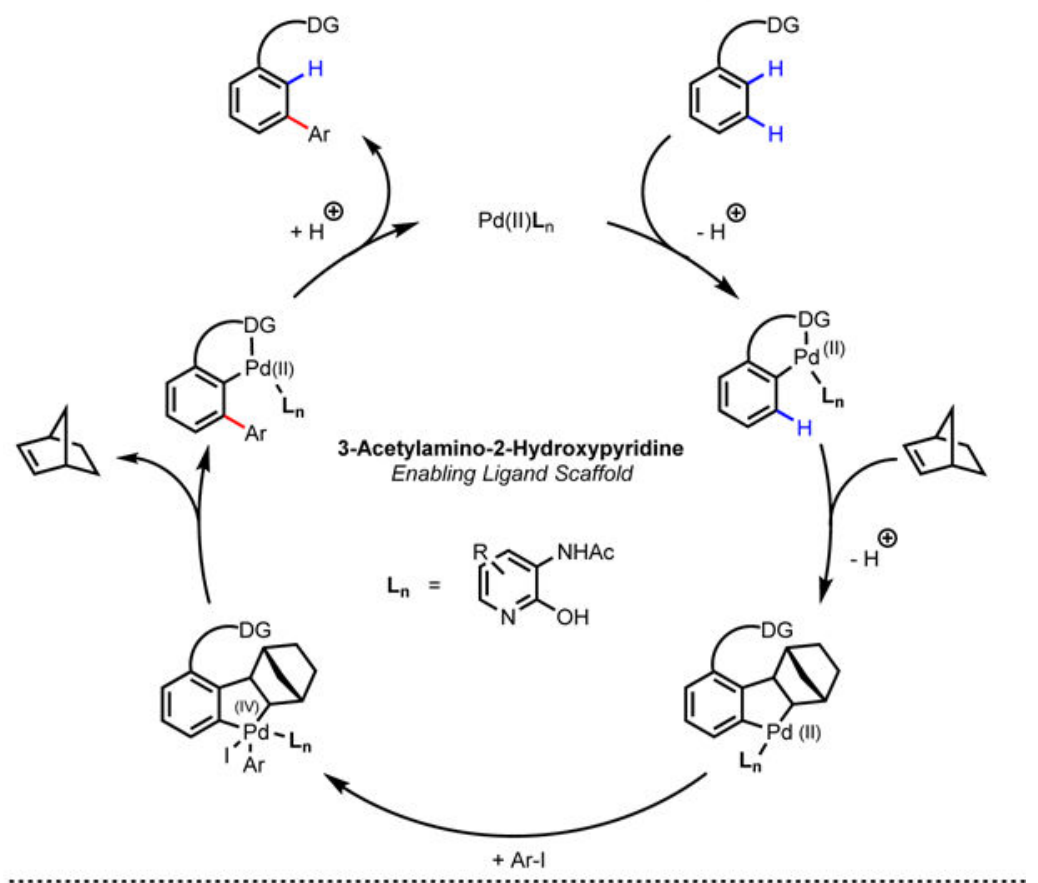
b. Design of 3-acylamino-2-hydroxypyridine ligands: analogy to MPAA ligands

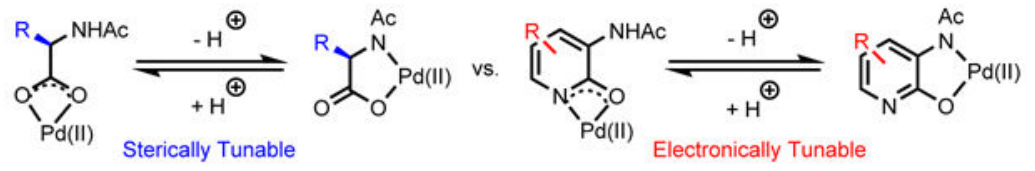

c. 3-acetylamino-2-hydroxypyridine enabled substrates and coupling partners
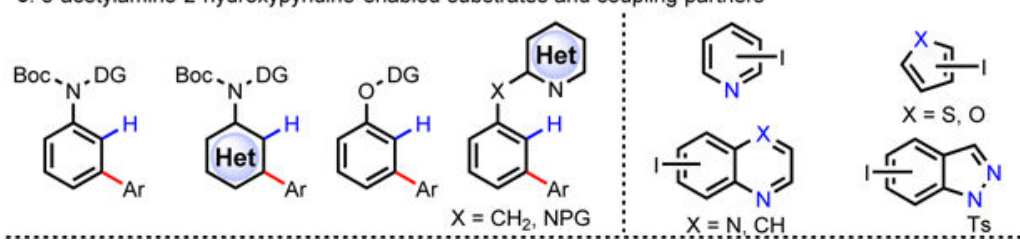

Figure 1.

Norbornene Mediated meta-C-H Arylation 


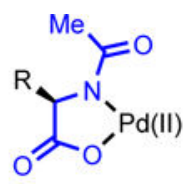

Computationally and experimentally supported active catalytic species for MPAA ligand promoted reactions.

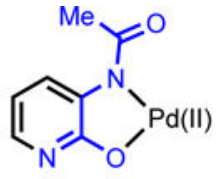

Does this play an analagous critical role in catalysis?

Figure 2.

Potential secondary coordination of 3-acylamino-2-hydroxypyridine ligands 
a. Functionalization of lenalidomide

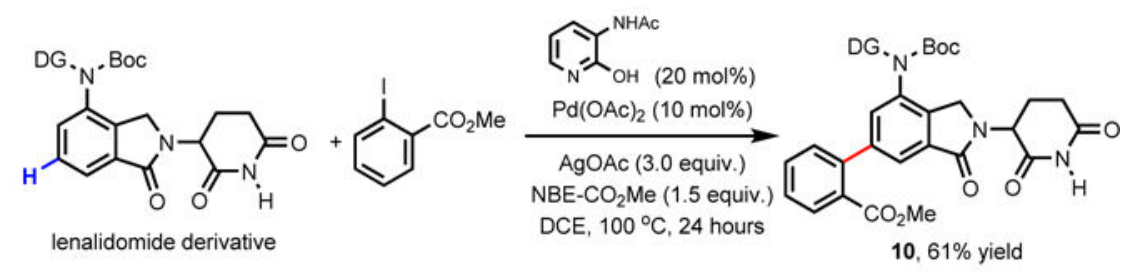

b. meta-Arylation of aniline without silver

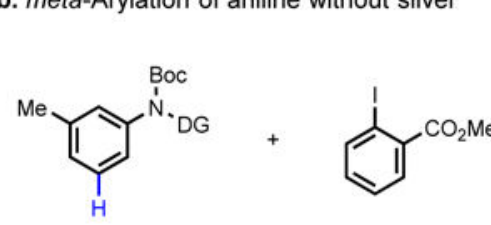

$1 \mathrm{a}, 1.43 \mathrm{~g}$

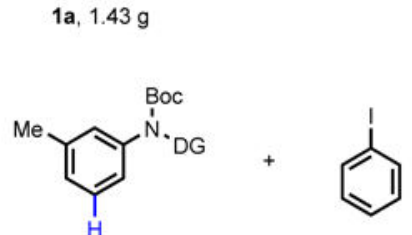

$1 \mathrm{a}$

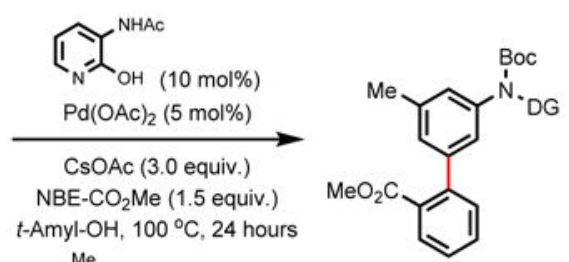
t-Amyl-OH, $100^{\circ} \mathrm{C}, 24$ hours

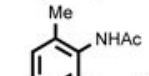

至 $(10 \mathrm{~mol} \%)$ $\mathrm{Pd}(\mathrm{OAC})_{2}(5 \mathrm{~mol} \%)$

$\mathrm{CsOAc}$ (3.0 equiv.) $\mathrm{NBE}-\mathrm{CO}_{2} \mathrm{Me}$ (1.5 equiv.) t-Amyl-OH, $100^{\circ} \mathrm{C}, 15$ hours then $\mathrm{Pd}(\mathrm{OAc})_{2}(5 \mathrm{~mol} \%)$ $\mathrm{CsOAc}$ ( 3.0 equiv.) t-Amyl-OH, 16 hours

$5 \mathrm{a}, 1.71 \mathrm{~g}, 87 \%$ yield

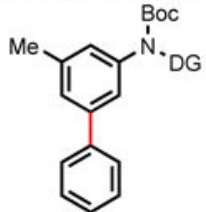

$9 a, 73 \%$ yield

Scheme 1.

Late Stage Functionalization and Removal of Silver. 


\section{Table 1}

Representative Ligand Evaluation ${ }^{a, b}$

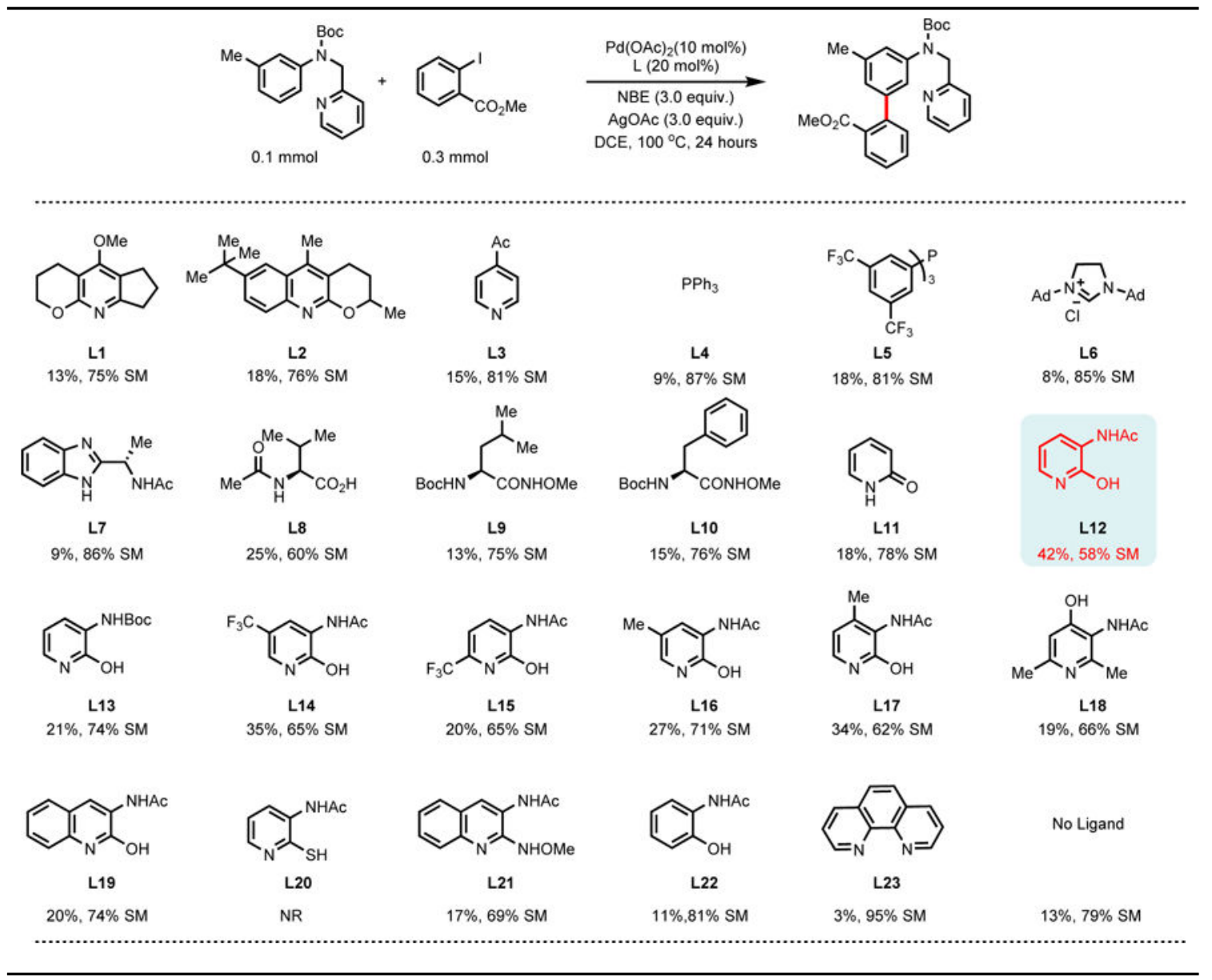

${ }^{a}$ Reaction conditions: substrate ( $\left.0.1 \mathrm{mmol}\right)$, methyl 2-iodobenzoate (2.0 equiv), $\mathrm{Pd}(\mathrm{OAc}) 2$ (10 mol\%), L (20 mol\%), $\mathrm{AgOAc}(3.0$ equiv), 2 norbornene (1.5 equiv), DCE $(0.5 \mathrm{~mL})$, air, $100^{\circ} \mathrm{C}, 24 \mathrm{~h}$.

$b_{\text {Yield was determined by }}{ }^{1} \mathrm{H}$ NMR using $\mathrm{CH}_{2} \mathrm{Br} 2$ as internal standard.; $\mathrm{DCE}=\mathrm{CLCH}_{2} \mathrm{CH}_{2} \mathrm{Cl}$. 
Table 2

Scope of Anilines, Aromatic Heterocyclic Amines, Phenols, and 2-Benzylheterocycles ${ }^{a, b}$

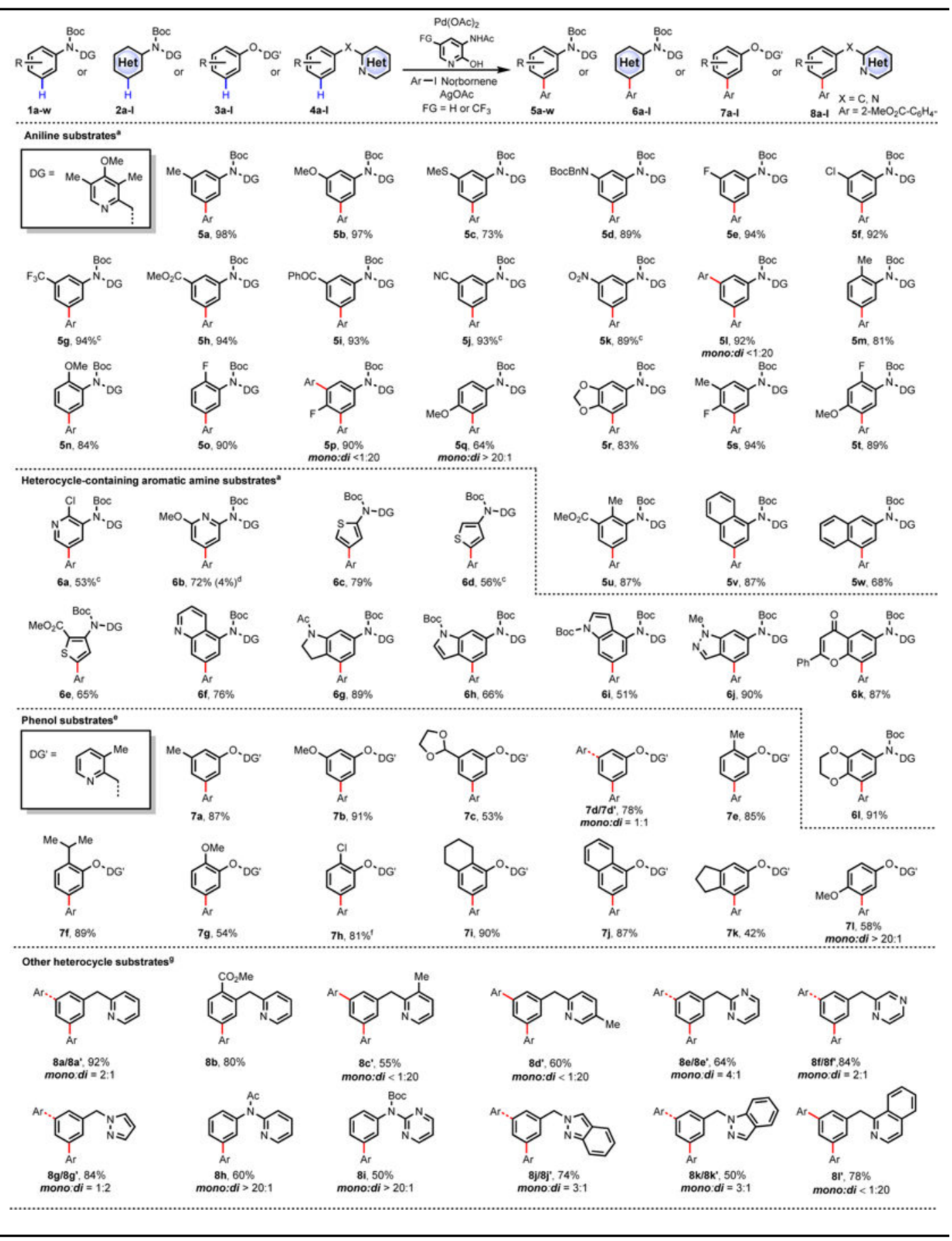

${ }^{a}$ Reaction conditions: 1 or 2 ( $\left.0.1 \mathrm{mmol}\right)$, methyl 2-iodobenzoate (2.0 equiv), Pd(OAc)2 (10 mol\%), L12 (20 mol\%), AgOAc (3.0 equiv), 2norbornene (1.5 equiv), $\mathrm{ClCH}_{2} \mathrm{CH}_{2} \mathrm{Cl}(0.5 \mathrm{~mL})$, air, $100{ }^{\circ} \mathrm{C}, 24 \mathrm{~h}$.

$b_{\text {Isolated yield. }}$ 
${ }^{c}$ Using NBE-CO2Me (1.5equiv.) instead of 2-norbornene.

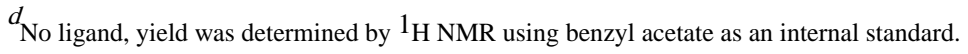

$e_{5}$ (0.2 mmol), methyl 2-iodobenzoate (3.0 equiv), $\mathrm{Pd}(\mathrm{OAc}) 2$ (10 mol\%), $\mathbf{L 1 4}$ (10 mol\%), $\mathrm{AgOAc}$ (3.0 equiv), $\mathrm{NBE}-\mathrm{CO}_{2} \mathrm{Me}(1.5 \mathrm{equiv}), \mathrm{CHCl}_{3}$ $(1.0 \mathrm{~mL})$, air, $100^{\circ} \mathrm{C}, 24 \mathrm{~h}$.

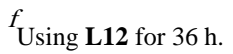

$g_{4}(0.1 \mathrm{mmol})$, methyl 2-iodobenzoate (3.0 equiv), $\mathrm{Pd}(\mathrm{OAc}) 2(10 \mathrm{~mol} \%), \mathbf{L 1 4}(20 \mathrm{~mol} \%), \mathrm{AgOAc}$ (3.0 equiv), 2-norbornene (1.5 equiv), $\mathrm{CHCl}_{3}$ $(1.0 \mathrm{~mL})$, air, $95{ }^{\circ} \mathrm{C}, 24 \mathrm{~h} ; \mathrm{NBE}-\mathrm{CO}_{2} \mathrm{Me}=$ methyl bicyclo[2.2.1]hept-2-ene-2-carboxylate. L12, FG = H. L14, FG = CF3, N.D. = Desired product was not detected. 


\section{Table 3}

Scope of Aryl Iodide Coupling Partners ${ }^{a, b}$

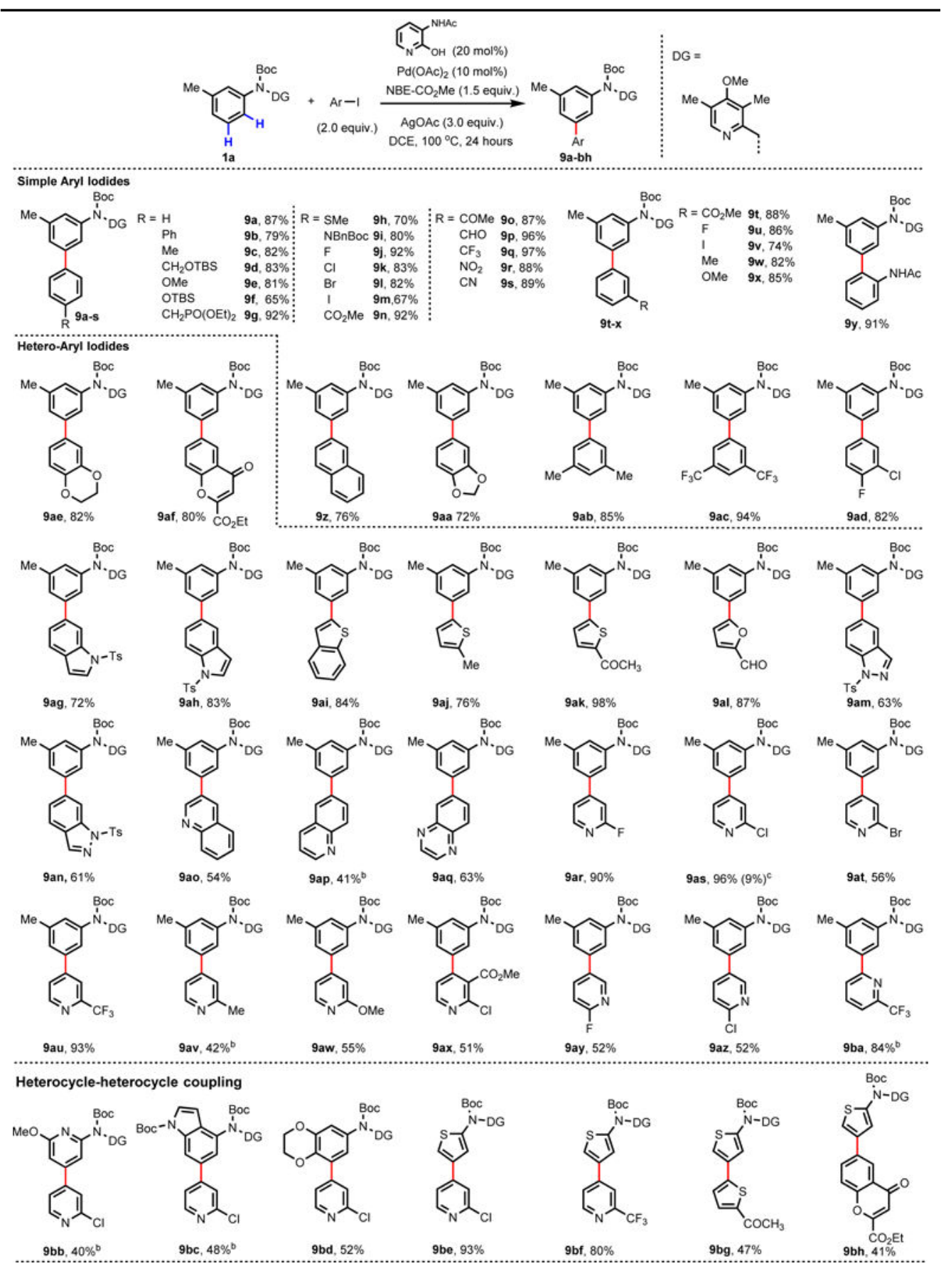

${ }^{a}$ Reaction conditions: 1a or 2 (0.1 mmol), Ar-I (0.2 mmol), Pd(OAc)2 (10 mol\%), L12 (20 mol\%), NBE-CO2Me (1.5 equiv.), AgOAc (3.0 equiv.), DCE $(0.5 \mathrm{~mL}), 100^{\circ} \mathrm{C}, 24$ hours.

$b_{\mathrm{Pd}(\mathrm{OAc}) 2}$ (20 $\left.\mathrm{mol} \%\right)$ and $\mathbf{L 1 2}(40 \mathrm{~mol} \%)$ were used.

J Am Chem Soc. Author manuscript; available in PMC 2017 July 27. 
${ }^{c}$ No L12, yield was determined by ${ }^{1} \mathrm{H}$ NMR using benzyl acetate as an internal standard.; NBE- $\mathrm{CO}_{2} \mathrm{Me}=$ methyl bicyclo[2.2.1]hept-2-ene-2carboxylate. $\mathrm{DCE}=\mathrm{ClCH}_{2} \mathrm{CH}_{2} \mathrm{Cl}$. 